\title{
Feasible Formations of Multi-Agent Systems ${ }^{1}$
}

\author{
Paulo Tabuada ${ }^{2} \quad$ George J. Pappas ${ }^{3} \quad$ Pedro Lima ${ }^{2}$ \\ ${ }^{2}$ Instituto de Sistemas e Robótica \\ Instituto Superior Técnico \\ 1049-001 Lisboa - Portugal \\ \{tabuada, pal\}@isr.ist.utl.pt \\ ${ }^{3}$ Department of EE \\ University of Pennsylvania \\ Philadelphia, PA 19104 \\ pappasg@ee.upenn.edu
}

\begin{abstract}
Formations of multi-agent systems, such as satellites and aircraft, require that individual agents satisfy their kinematic equations while constantly maintaining inter-agent constraints. In this paper, we develop a systematic framework for studying formations of multiagent systems. In particular, we consider undirected formations for centralized formations and directed formations for decentralized formations. In each case, we determine differential geometric conditions that guarantee formation feasibility given the individual agent kinematics. Our framework also enables us to extract a smaller control system that describes the formation kinematics while maintaining all formation constraints.
\end{abstract}

\section{Introduction}

Advances in communication and computation have enabled the distributed control of multi-agent systems. This philosophy has resulted in next generation automated highway systems [9], coordination of aircraft in future air traffic management systems [8], as well as formation flying aircraft, satellites, and multiple mobile robots $[2,3,7,4]$.

The control of multiple homogeneous or heterogeneous agents raises fundamental questions regarding the formation control of a group of agents. Multi-agent formations require individual agents to satisfy their kinematics while constantly satisfying inter-agent constraints. In typical leader-follower formations, the leader has the responsibility of guiding the group, while the followers have the responsibility of maintaining the inter-agent formation. Distributing the group control tasks to individual agents must be compatible with the control and sensing capabilities of the individual agents. As

\footnotetext{
${ }^{1}$ This work was performed while the first author was visiting the University of Pennsylvania. This research is partially supported by DARPA under grant N66001-99-C-8510, the University of Pennsylvania Research Foundation, and by Fundação para a Ciência e Tecnologia under grant PRAXIS XXI/BD/18149/98.
}

the inter-agent dependencies get more complicated, a systematic framework for controlling formations is vital.

In this paper, we propose a framework for formation control of multi-agent systems. Formations are modeled using formation graphs which are graphs whose nodes capture the individual agent kinematics, and whose edges represent inter-agent constraints that must be satisfied. A similar approach has been proposed in [4]. We assume kinematic models for each agent described by drift free control systems. This class of systems is rich enough to capture holonomic, nonholonomic, or underactuated agents. Two distinct types of formations are considered : undirected formations and directed formations.

In undirected formations each agent is equally responsible for maintaining the formation. For each edge constraining two agents of the formation graph, both agents cooperate in order to satisfy the constraint. Undirected formations therefore present a more centralized approach to the formation control problem as communication between agents is, in general, necessary. In directed formations, for each edge constraining two agents, only one of the agents (the follower) is responsible for maintaining the constraint. Directed formations, therefore, represent a more decentralized solution to the formation control problem.

In this paper, we focus on the feasibility problem: Given the kinematics of several agents along with the inter-agent constraints, determine whether there exist agent trajectories that maintain the constrains. For both directed and undirected formations we obtain differential-geometric conditions that determine formation feasibility. When such conditions are verified the formation contral abstraction problem is then considered: Given a feasible formation, extract a smaller control system that maintains formations along its trajectories. The extracted control system allows to control the formation as a single entity, therefore being well suited for higher levels of control.

The structure of this paper is as follows: In Section 2 we 
define the notion of a formation graph. In Section 3 we consider the feasibility problem for undirected formations, whereas in Section 4 we consider it for directed formations. Finally, Section 5 describes many interesting directions of further research.

\section{Formation Graphs}

We assume the reader is familiar with various differential geometric concepts at the level of [1]. Consider $n$ heterogeneous agents with states $x_{i}(t) \in M_{i}$, $i=1, \ldots, n$ whose kinematics are defined by drift free controlled distributions on manifolds $M_{i}$ as:

$$
\begin{aligned}
\Delta_{i} & : M_{i} \times U_{i} \rightarrow T M_{i} \\
\Delta_{i} & =\sum_{j} X_{j} u_{j}
\end{aligned}
$$

where $U_{i}$ is the control space, and the vector fields $X_{j}$ form a basis for the distribution. The controlled distributions are general enough to model nonholonomy and underactuation. A distribution $\Delta_{i}$ can be equivalently defined by its annihilating codistribution $\omega_{K_{i}}$ defined as [5]:

$$
\omega_{K_{i}}=\left\{\alpha \in T^{*} M_{i} \mid \alpha(\Delta)=0\right\}
$$

The formation of a set of agents is defined by the formation graph which completely describes individual agent kinematics and global inter-agent constrains.

Definition 2.1 (Formation Graph) $A$ formation graph $F=(V, E, C)$ consists of:

- A finite set $V$ of vertices who's cardinality is equal to the number of agents. Each vertex $v_{i}$ : $M_{i} \times U_{i} \rightarrow T M_{i}$ is a distribution $\Delta_{i}$ modeling the kinematics of each individual agent as described in (1).

- A binary relation $E \subset V \times V$ representing a link between agents.

- A family of constraints $C$ indexed by the set $E$, $C=\left\{c_{e}\right\}_{e \in E}$. For each edge $e=\left(v_{i}, v_{j}\right), c_{e}$ is a possibly time varying function $c_{e}\left(x_{i}, x_{j}, t\right)=0$ describing the $\phi(e)$ independent constraints between vertices $v_{i}$ and $v_{j}$. For a generic edge $e=\left(v_{i}, v_{j}\right), c_{e}$ is mathematically defined as $c_{e}$ : $M_{i} \times M_{j} \times \mathbb{R} \rightarrow \mathbb{R}^{\phi(e)}, \phi(e) \in \mathbb{N} \quad \forall_{e \in E}$.

Two different types of formation graphs will be considered: undirected formations where $(V, E)$ will be an undirected graph and directed formations where $(V, E)$ will be a directed graph. In undirected formations, for each edge $e=\left(v_{i}, v_{j}\right)$ both agents are equally responsible for maintaining the associated constraint $c_{e}$, whereas for directed formations the constraint $c_{e}$ must be maintained by agent $i$. At this point no further structure is assumed on the set $E$, additional structure will be explicitly mentioned when needed throughout the paper.

In this paper, we focus on the formation feasibility problem, more precisely:

Problem 2.2 Given a formation graph $F=(V, E, C)$ determine whether there are solutions $x_{i}(t)$ of all agent kinematics (1) that maintain the constraints $c_{e}$ for all $e \in E$.

We will solve Problem 2.2 for both undirected and directed formations. In case the formation is feasible, a new problem immediately emerges, the extraction of a formation control abstraction which characterizes the solution space of Problem 2.2 :

Problem 2.3 Given a feasible formation graph $F=$ $(V, E, C)$, extract a smaller control system that maintains formation for all values of its control inputs.

Problem 2.3 will also be solved for both the undirected and the directed cases.

\section{Undirected Formations}

\subsection{Feasibility}

In undirected formations each agent is equally responsible for maintaining constraints. Because of this property it will be useful to collect all agent kinematics and constraints on a single manifold:

$$
M=\prod_{i=1}^{n} M_{i}
$$

Given an element $x$ of $M$ the canonical projection on the $i$ th agent:

$$
\pi_{i}: M \rightarrow M_{i}
$$

allow us to denote the state of the individual agents by $x_{i}=\pi_{i}(x)$. The formation kinematics is obtained by appending individual kinematics through direct sum, that is:

$$
\begin{aligned}
& \Delta: M \times U \rightarrow T M \\
& \Delta=\oplus_{i=1}^{n} \Delta_{i}
\end{aligned}
$$

where $U$ is taken to be $U=\prod_{i=1}^{n} U_{i}$. To lift the individual constraints $c_{e}$ from $M_{i} \times M_{j} \times \mathbb{R}, i, j \in\{1,2, \ldots, n\}$ 
to the group manifold $M$ we define $\mathcal{C}_{e}$ by:

$$
\begin{aligned}
\mathcal{C}_{e} & : \quad M \times \mathbb{R} \rightarrow \mathbb{R}^{\phi(e)} \\
\mathcal{C}_{e}(x, t) & =c_{e}\left(\pi_{i}(x), \pi_{j}(x), t\right)
\end{aligned}
$$

Formation feasibility requires that the constraints are satisfied along the formation trajectories, more precisely:

$$
\frac{d}{d t} \mathcal{C}_{e}=\mathcal{L}_{X} \mathcal{C}_{e}+\frac{\partial \mathcal{C}_{e}}{\partial t}=0 \quad \forall \forall_{e \in E}
$$

When $\mathcal{C}_{e}$ is vector valued we consider that the Lie derivate of $\mathcal{C}_{e}$ along $X$ will be given by $\mathcal{L}_{X} \mathcal{C}_{e}=$ $\left[\mathcal{L}_{X} \mathcal{C}_{e}^{1} \mathcal{L}_{X} \mathcal{C}_{e}^{2} \ldots \mathcal{L}_{X} \mathcal{C}_{e}^{\phi(e)}\right]^{T}$. To develop a single mathematical object that will allow us to check for feasibility we will adopt a differential forms approach instead of working directly with the vector fields. By defining the exterior derivative of $\mathcal{C}_{e}$ as $\mathrm{d} \mathcal{C}_{e}=\left[\begin{array}{lll}\mathrm{d}_{e}^{1} & \mathrm{~d} \mathcal{C}_{e}^{2} & \ldots \mathrm{d} \mathcal{C}_{e}^{\phi(e)}\end{array}\right]^{T}$ equation (7) can be written as $\left.\mathrm{d}_{e}\right|_{t}(X)=-\frac{\partial}{\partial t} \mathcal{C}_{e}$, where we have denoted by $\left.\mathrm{d} \mathcal{C}_{e}\right|_{t}$ the exterior derivative of $\mathcal{C}_{e}$ for fixed $t$. If we now consider an enumeration $\{1,2, \ldots, m\}$ of the edges set $E$ and define the following vector valued forms ${ }^{1}$ :

$$
\omega_{F}=\left[\begin{array}{c}
\left.\mathrm{d} \mathcal{C}_{1}\right|_{t} \\
\left.\mathrm{~d} \mathcal{C}_{2}\right|_{t} \\
\vdots \\
\left.\mathrm{d} \mathcal{C}_{m}\right|_{t}
\end{array}\right] \quad T_{F}=-\left[\begin{array}{c}
\frac{\partial \mathcal{C}_{1}}{\partial t} \\
\frac{\partial \mathcal{C}_{2}}{\partial t} \\
\vdots \\
\frac{\partial \mathcal{C}_{m}}{\partial t}
\end{array}\right]
$$

we can express equation (7) as:

$$
\omega_{F}(X)=T_{F}
$$

The kinematics can also be modeled as differential forms by using the annihilating codistributions. This lead us to define a single codistribution $\omega_{K}$ modeling the kinematics of all formation agents as:

$$
\omega_{K}=\left[\begin{array}{llll}
\omega_{K_{1}} & \omega_{K_{2}} & \cdots & \omega_{K_{n}}
\end{array}\right]^{T}
$$

Solutions of equation (9) represent vector fields that maintain formation while solutions of equation (10) satisfy the kinematics. Therefore by merging both objects into:

$$
\Omega=\left[\begin{array}{l}
\omega_{F} \\
\omega_{K}
\end{array}\right] \quad T=\left[\begin{array}{c}
T_{F} \\
0
\end{array}\right]
$$

we can check for formation feasibility in a single equation:

$$
\Omega(X)=T
$$

The previous discussion leads to the following solution of Problem 2.2:

\footnotetext{
${ }^{1}$ This definition is independent of the chosen enumeration as can be easily verified.
}

Proposition 3.1 An undirected formation is feasible iff equation (12) has solutions, equivalently iff $T$ belongs to the range of $\Omega$.

Corollary 3.2 (Time-Invariant Case) If the formation constraints $C$ are time-invariant then the undirected formation is feasible iff $\Omega$ (thought as a pointwise linear map between vector spaces) is not of full rank.

A solution of equation $\Omega(X)=T$ specifies the motion of each individual agent. When more than one independent solution exists, a change in the direction of a single agent may require that all other agents also change their actions to maintain formation. This shows that, in general, solutions for undirected formations are centralized and require inter-agent communication for their implementation.

\subsection{Group Abstraction}

Whenever more then one independent solutions exist, the solution space of equation $\Omega(X)=T$ can be used to extract a smaller control system that will preserve the formation along its trajectories. This new control system is an abstraction that hides away low-level control necessary to maintain the formation and can be used in higher levels of control. Since the solution space is in general an affine space the new control system will also be affine in the control. Let $K_{p}$ be a particular solution of equation (12), Problem 2.3 is therefore solved by the new control system:

$$
\Delta_{G}=K_{p}+\operatorname{Ker}(\Omega)
$$

If we now denote by $\left\{K_{1}, K_{2}, \ldots, K_{k}\right\}$ a basis for the kernel of $\Omega$ we can rewrite (13) in a more usual form as:

$$
\Delta_{G}=K_{p}+\sum_{j=1}^{k} K_{j} u_{j}
$$

In the time-independent case we recover linearity of the abstracted control system since we can chose $K_{p}=0$. The centralized nature of the problem is also reflected on the control abstraction. When one or more of the control inputs $u_{i}$ are used, inter-agent cooperation is necessary to implement the new direction of motion since each vector $K_{j}$ specifies the motion for all formation agents.

In addition to using the above abstracted system to control the formation, one can also guide the formation by appending a virtual vertex $v_{0}$ defining the reference trajectory and several edges specifying how the reference should be followed by the formation. In particular consider a feasible formation graph $F=(V, E, C)$ and let $V^{\prime}$ be a singleton containing the vertex $v_{0}$ : $\mathbb{R} \rightarrow T M_{0}, v_{0}=\frac{d}{d t} x_{0}(t)$. This vertex is connected 
to the remaining formation by the additional edge set $E^{\prime}=\cup_{i \in I}\left\{\left(v_{0}, v_{i}\right)\right\}$, where $I \subseteq V$ is a subset of all the vertices indices. Associated with each vertex we have the constraints $C^{\prime}=\left\{c_{e}^{\prime}\right\}_{e^{\prime} \in E^{\prime}}$ and we can define a new formation graph given by $F^{\prime}=\left(V^{\prime} \cup V, E^{\prime} \cup E, C^{\prime} \cup C\right)$. Once again it is necessary to ensure that the feasible formation is capable of maintaining the reference constraints by applying Proposition 3.1 to formation graph $F^{\prime}$.

Note that this construction is general enough to encompass traditional formations such as: leader-follower by superimposing the virtual vertex onto an existing one or placing references on the formation centroid $[4,7]$. It also allows some other interesting possibilities such as connecting a disconnected feasible formation graph by the reference constraints, that is several independent formations following a single reference.

Example: Consider two planar robots evolving on $M_{i}=\mathbb{R}^{2} \times \mathbb{S}^{1} i=1,2$, parameterized by $\left(x_{i}, y_{i}, \theta_{i}\right)$, $\theta_{i} \in\left[0,2 \pi\left[, x_{i}, y_{i} \in \mathbb{R}\right.\right.$. Robot 1 is nonholonomic, therefore only motions along the direction where it is pointed to are allowed while robot 2 is holonomic being able to move in any direction. The two robots are described by the following controlled distributions:

$$
\begin{aligned}
& \Delta_{1}=X_{1}^{1} u_{1}+X_{2}^{1} u_{2} \\
& \Delta_{2}=X_{1}^{2} u_{1}+X_{2}^{2} u_{2}+X_{3}^{2} u_{3}
\end{aligned}
$$

where the vectors $X_{1}, X_{2}$ and $X_{3}$ are defined as:

$$
X_{1}^{i}=\left[\begin{array}{l}
0 \\
0 \\
1
\end{array}\right] \quad X_{2}^{i}=\left[\begin{array}{c}
\cos \theta_{i} \\
\sin \theta_{i} \\
0
\end{array}\right] \quad X_{3}^{i}=\left[\begin{array}{c}
-\sin \theta_{i} \\
\cos \theta_{i} \\
0
\end{array}\right]
$$

Equivalently the kinematics of robot 1 and 2 can be collectively modeled by the following form:

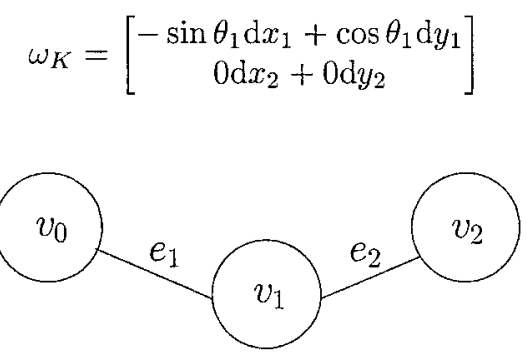

Figure 1: Graph used to specify the undirected formation.

The desired formation is presented on Figure 1. Vertex $v_{0}$ is a virtual node associated with the reference trajectory given by $(\dot{a}(t), \dot{b}(t))$. The constraints associated with edge $e_{1}$ are given by $c_{e_{1}}=\left[\begin{array}{ll}x_{0}-x_{1} & y_{0}-y_{1}\end{array}\right]^{T}$, therefore the position of vertex $v_{0}$ will be the same as the position of vertex $v_{1}$, but no constraints exist on the orientation. The constraints associated with edge $e_{2}$ are $c_{e_{2}}=\left[\begin{array}{lll}x_{1}-x_{2}-k_{x} & y_{1}-y_{2}-k_{y} & \theta_{1}-\theta_{2}\end{array}\right]^{T}$ for some positive offsets $k_{x}$ and $k_{y}$. These constraints require that both agents perform equal trajectories translated by the offsets $k_{x}$ and $k_{y}$. From the constraints we compute the form $\omega_{F}$ and the vector $T_{F}$ :

$$
\omega_{F}=\left[\begin{array}{c}
-\mathrm{d} x_{1} \\
-\mathrm{d} y_{1} \\
\mathrm{~d} x_{1}-\mathrm{d} x_{2} \\
\mathrm{~d} y_{1}-\mathrm{d} y_{2} \\
\mathrm{~d} \theta_{1}-\mathrm{d} \theta_{2}
\end{array}\right] \quad T_{F}=\left[\begin{array}{c}
-\dot{a}(t) \\
-\dot{b}(t) \\
0 \\
0 \\
0
\end{array}\right]
$$

Constructing the form $\Omega$ and the vector $T$ from the above forms we easily see that $T$ belongs to the range of $\Omega$, as long as $\dot{b}(t) \cos \theta_{1}-\dot{a}(t) \sin \theta_{1}=0$ (meaning that robot 1 must be aligned with the reference velocity) therefore the formation is feasible. If we swap the location of the robots, the new form $\omega_{K}$ will be given by:

$$
\omega_{K}=\left[\begin{array}{c}
0 \mathrm{~d} x_{1}+0 \mathrm{~d} y_{1} \\
-\sin \theta_{2} \mathrm{~d} x_{2}+\cos \theta_{2} \mathrm{~d} y_{2}
\end{array}\right]
$$

and the equation $\Omega(X)=T$ has solutions as long as robot 1 is aligned with the reference velocity and since both robots must share the same orientation, robot 2 must also be aligned with the reference velocity. Both undirected formations are feasible but this is not the case when dealing with directed formations as we shall see next.

\section{Directed Formations}

Another important class of formations can be modeled by directed graphs. A directed graph assigns responsibilities to the formation members in an asymmetric way. For each edge $e=\left(v_{i}, v_{j}\right)$ agent $i$ is responsible for maintaining the constraints $c_{e}$, while agent $j$ is not affected by the constraint of the edge.

We will assume through the remaining section that a directed formation graph is a directed acyclic graph. As a consequence all directed formations will have at least one leader. This assumption will allow the recursive procedures to start on the leaders and to terminate since there are no cycles. Cyclic formation graphs, although important, will be discussed separately [6]. We will also consider that the formation constraints are time independent for simplicity of presentation although the results can easily be extended to time-varying constraints.

Although in the undirected case we were able to lift the constraints and individual agents kinematics to a larger manifold $M$, the same approach will not be possible for the directed case since only one agent is responsible for satisfying the constraints associated with an edge. More precisely, given an edge $e=\left(v_{i}, v_{j}\right)$ the 
time derivative of its associated constraints $c_{e}$ can be decomposed as:

$$
\frac{d c_{e}}{d t}=\mathcal{L}_{X_{i}} c_{e}+\mathcal{L}_{X_{j}} c_{e}
$$

Feasibility requires that $\frac{d c_{e}}{d t}=0$, however only $X_{i}$ can be chosen to ensure feasibility. In view of this we will follow a similar approach to the undirected case, but in a recursive formulation. This requires the following operators:

Definition 4.1 (Post and Pre) Let $F=(V, E, C)$ be a directed formation graph. The Post operator is defined by

$$
\text { Post: } \begin{aligned}
V & \rightarrow 2^{V} \\
v_{i} & \mapsto\left\{v_{j} \in V:\left(v_{i}, v_{j}\right) \in E\right\}
\end{aligned}
$$

Similarly, the Pre operator is defined as:

$$
\begin{aligned}
\text { Pre: } V & \rightarrow 2^{V} \\
v_{i} & \mapsto\left\{v_{j} \in V:\left(v_{j}, v_{i}\right) \in E\right\}
\end{aligned}
$$

Intuitively, Post $\left(v_{i}\right)$ will return the agents that are leading agent $i$, while $\operatorname{Pre}\left(v_{i}\right)$ will return all the agents that are following agent $i$. Post and Pre extend to sets of vertices in the natural way, $\operatorname{Post}(P)=\cup_{p \in P} \operatorname{Post}(p)$ and $\operatorname{Pre}(P)=\cup_{p \in P} \operatorname{Pre}(p)$.

Definition 4.2 (Leaders) A vertex $v_{i}$ is called a leader iff $\operatorname{Post}\left(v_{i}\right)=\varnothing$.

We shall abuse notation a represent the distribution $\Delta_{i}$ defining the kinematics of agent $v_{i}$ by $\Delta\left(v_{i}\right)$ and for the set of agents $\operatorname{Post}\left(v_{i}\right), \Delta\left(\operatorname{Post}\left(v_{i}\right)\right)=\oplus_{p \in \operatorname{Post}\left(v_{i}\right)} \Delta(p)$ defined over $\Pi_{p \in \operatorname{Post}\left(v_{i}\right)} M_{p}$. Similarly to the undirected case we define the following objects for each agent $i$ :

$$
\omega_{F}^{i}=\left[\begin{array}{c}
\left.\mathrm{d} c_{1}\right|_{x_{j}} \\
\left.\mathrm{~d} c_{2}\right|_{x_{j}} \\
\vdots \\
\left.\mathrm{d} c_{m}\right|_{x_{j}}
\end{array}\right] \quad \omega_{F}^{j}=-\left[\begin{array}{c}
\left.\mathrm{d} c_{1}\right|_{x_{i}} \\
\left.\mathrm{~d} c_{2}\right|_{x_{i}} \\
\vdots \\
\left.\mathrm{d} c_{1}\right|_{x_{i}}
\end{array}\right]
$$

where $\{1,2, \ldots m\}$ is an enumeration of the edges set between agent $i$ and its leaders $\left(\operatorname{Post}\left(v_{i}\right)\right)$. These vector valued differential forms allow us to write equation (20) as:

$$
\omega_{F}^{i}\left(X_{i}\right)=\omega_{F}^{j}\left(X_{J}\right)
$$

which is to be considered only for $X_{i} \in \Delta\left(v_{i}\right)$ and $X_{J} \in$ $\Delta\left(\operatorname{Post}\left(v_{i}\right)\right)$. Instead of restricting the $X_{i}$ 's to $\Delta\left(v_{i}\right)$ we can incorporate the kinematic restrictions directly into equation (24) by defining:

$$
\Omega^{i}=\left[\begin{array}{l}
\omega_{F}^{i} \\
\omega_{K}^{i}
\end{array}\right] \quad \Omega^{j}=\left[\begin{array}{c}
\omega_{F}^{j} \\
0
\end{array}\right]
$$

where $\omega_{K}^{i}$ is the vector valued form annihilating agent $i$ kinematic distribution $\Delta\left(v_{i}\right)$. Equation (20) can now be further modified to the following form.

$$
\Omega^{i}\left(X_{i}\right)=\Omega^{j}\left(X_{J}\right) \quad \forall_{X_{J} \in \Delta\left(\operatorname{Post}\left(v_{i}\right)\right)}
$$

This motivates the following result analogous to the undirected case:

Proposition $4.3 \mathrm{~A}$ directed formation is feasible iff equation (26) has solutions for each agent $i$ in the formation. Equivalently iff the range of $\left.\Omega^{j}\right|_{\Delta\left(\operatorname{Post}\left(v_{i}\right)\right)}$ is contained in the range of $\Omega^{i}$ for each agent $i$.

Since Proposition 4.3 must be true for each agent, an algorithm can be constructed to determine feasibility. Let $L \subset V$ be a set of leaders and denote by $\left(\Omega^{i}\right)^{-1}(X)$ the set of preimages of $X$ under $\Omega^{i}$ and by $\mathcal{R}(S)$ the range of operator $S$.

\section{Algorithm 1 (Directed Feasibility)}

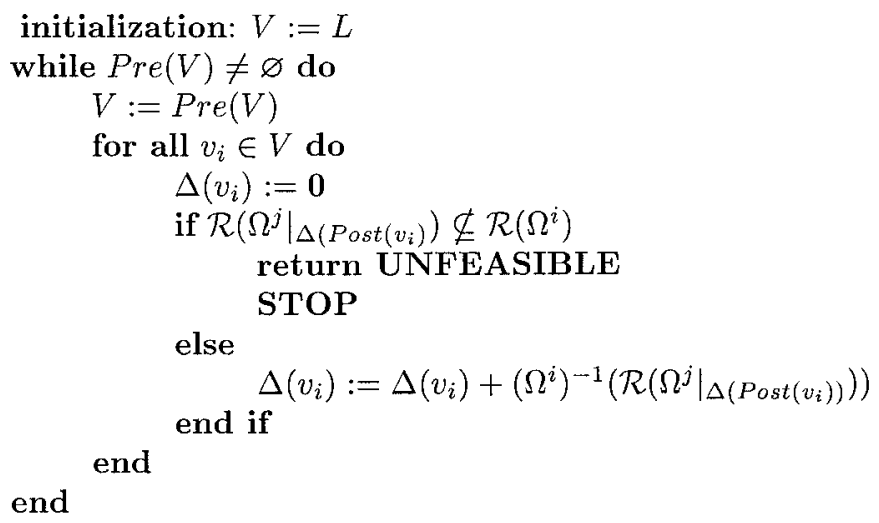

All the computations in the algorithm can be performed using basis vector fields for the distributions and since there are no cycles in the algorithm we have the following result:

\section{Theorem 4.4 (Directed Formation Feasibility)} Let $F=(V, E, C)$ be an acyclic, directed formation graph. Algorithm 1 terminates in a finite number of steps and returns:

- Unfeasible if the formation is not feasible.

- A distribution per agent specifying the available directions to maintain formation if the formation is feasible.

Example: Consider the formation graphically displayed in Figure 2, where agent 1 and agent 2 are as in the previous example. Similarly we associate the constraint $c_{e}=\left[\begin{array}{lll}x_{1}-x_{2}-k_{x} & y_{1}-y_{2}-k_{y} & \theta_{1}-\theta_{2}\end{array}\right]^{T}$ 


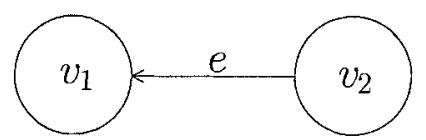

Figure 2: Graph used to specify the directed formation.

to edge $e=\left(v_{2}, v_{1}\right)$. To determine feasibility of this directed formation one has to compute:

$$
\omega_{F}^{2}=\left[\begin{array}{l}
-\mathrm{d} x_{2} \\
-\mathrm{d} y_{2} \\
-\mathrm{d} \theta_{2}
\end{array}\right] \quad \omega_{F}^{1}=\left[\begin{array}{l}
-\mathrm{d} x_{1} \\
-\mathrm{d} y_{1} \\
-\mathrm{d} \theta_{1}
\end{array}\right]
$$

and also:

$$
\Omega^{2}=\left[\begin{array}{c}
-\mathrm{d} x_{2} \\
-\mathrm{d} y_{2} \\
-\mathrm{d} \theta_{2} \\
0
\end{array}\right] \quad \Omega^{1}=\left[\begin{array}{c}
-\mathrm{d} x_{1} \\
-\mathrm{d} y_{1} \\
-\mathrm{d} \theta_{1} \\
-\sin \theta_{1} \mathrm{~d} x_{1}+\cos \theta_{1} \mathrm{~d} y_{1}
\end{array}\right]
$$

Feasibility now requires that $\mathcal{R}\left(\left.\Omega^{1}\right|_{\Delta\left(\operatorname{Post}\left(v_{2}\right)\right)}\right) \subseteq$ $\mathcal{R}\left(\Omega^{2}\right)$, but since $\operatorname{Post}\left(v_{2}\right)=v_{1}$ and agent $v_{1}$ has no kinematic constraints, we get $\mathcal{R}\left(\left.\Omega^{1}\right|_{\Delta\left(\operatorname{Post}\left(v_{2}\right)\right)}\right)=$ $\mathcal{R}\left(\Omega^{1}\right)$. From this we see clearly that the conditions of Theorem 4.4 are not fulfilled and the directed formation is not feasible. Maintaining the formation requires a cooperative effort from agent $v_{1}$ to cope with agent $v_{2}$ nonholonomic restrictions. However if we change the position of the robots in the formation we render the formation feasible. In this situation the new forms are given by:

$$
\Omega^{2}=\left[\begin{array}{c}
\mathrm{d} x_{2} \\
\mathrm{~d} y_{2} \\
\mathrm{~d} \theta_{2} \\
\sin \theta_{2} \mathrm{~d} x_{2}+\cos \theta_{2} \mathrm{~d} y_{2}
\end{array}\right] \quad \Omega^{1}=\left[\begin{array}{c}
\mathrm{d} x_{1} \\
\mathrm{~d} y_{1} \\
\mathrm{~d} \theta_{1} \\
0
\end{array}\right]
$$

and the inclusion $\mathcal{R}\left(\left.\Omega^{1}\right|_{\Delta\left(\operatorname{Post}\left(v_{2}\right)\right)}\right) \subseteq \mathcal{R}\left(\Omega^{2}\right)$ is satisfied, meaning that formation feasibility is achieved. This shows, in particular, that one can break the global undirected solution into local ones, for e.g. agent $v_{1}$ does not need to know that it is being followed. From an implementation point of view this means that agent $v_{1}$ control laws are independent from agent $v_{2}$ state.

When a directed formation is feasible the formation control abstraction is trivially taken as the control systems of the leaders. Contrary to the undirected case this abstraction does not allow direct control over each individual agent. Control is exerted on the leaders that indirectly control the formation through the interagents links.

\section{Conclusions}

In this paper we have proposed a general framework for determining feasibility of formations. Two differ- ent types of formations were considered: undirected formations were inter-agent cooperation is required to maintain formation and directed formations were control responsibilities are distributed between the agents. Conditions were developed to determine formation feasibility for the two type of formations considered and a control abstraction for the group was also extracted to model the formation as single object in higher control layers.

When a directed formation is not feasible it may still be possible to extract a feasible formation by reducing the degrees of freedom that cannot be handled by the followers. This direction of research will be addressed in forthcoming publications as well as considering directed formation graphs with possible cycles.

\section{References}

[1] R. Abraham, J. Marsden, and T. Ratiu. Manifolds, Tensor Analysis and Applications. Applied Mathematical Sciences. Springer-Verlag, 1988.

[2] T. Balch and R. Arkin. Behavior-based formation control for multirobot systems. IEEE Transactions on Robotics and Automation, 14(12), 1998.

[3] R.W. Beard, J.Lawton, and F.Y. Hadaegh. A coordination architecture for spacecraft formation control. IEEE Transactions on Control Systems Technology, 2000. To appear.

[4] J. Desai, V. Kumar, and J. Ostrowski. Control of changes in formation of multi-robot teams. In Proceedings of the 1999 International Conference on Robotics and Automation, Detroit 1998.

[5] George J. Pappas, John Lygeros, Dawn Tilbury, and Shankar Sastry. Exterior differential systems in control and robotics. In J. Baillieul, S. Sastry, and H. Sussmann, editors, Essays on Mathematical Robotics, volume 104 of IMA Volumes in Mathematics and its Applications, pages 271-372. Springer Verlag, New York, 1998.

[6] Paulo Tabuada, George J. Pappas, and Pedro Lima. Cyclic directed formations of multi-agent systems. European Control Conference, Porto, Portugal, September 2001. Submitted.

[7] Kar-Han Tan and M. Anthony Lewis. Virtual structures for high-precision cooperative mobile robot control. Autonomous Robots, 4(4):387-403, October 1997.

[8] C. Tomlin, G. J. Pappas, and S. Sastry. Conflict resolution for air traffic management : A study in multi-agent hybrid systems. IEEE Transactions on Automatic Control, 43(4):509-521, April 1998.

[9] P. Varaiya. Smart cars on smart roads: problems of control. IEEE Transactions on Automatic Control, 38(2):195-207, 1993. 